Pesq. Vet. Bras. 29(4):333-338, abril 2009

\title{
Aspectos morfológicos da ultra-sonografia hepática de ovinos $^{1}$
}

\author{
Pedro B. Néspoli2*, Valentim A. Gheller ${ }^{3}$, German A. B. Mahecha ${ }^{4}$, Douglas K. \\ Godoy de Araújo ${ }^{5}$, Gilberto L. Macedo Júnior ${ }^{6}$ e Angela I. Bordin ${ }^{7}$
}

\begin{abstract}
Néspoli P.B, Gheller V.A., Mahecha G.A.B., Godoy de Araújo D.K., Gillberto L. Macedo G.L.J. \& Bordin A.I. 2009. [Morphologic aspects of hepatic ultrasonography in sheep.] Aspectos morfológicos da ultra-sonografia hepática de ovinos. Pesquisa Veterinária Brasileira 29(4):333-338. Departamento de Clínica Médica Veterinária, Faculdade Agronomia e Medicina Veterinária, Universidade Federal de Mato Grosso, Av. Fernando Corrêa da Costa s/n, Coxipó, Cuiabá, MT 78060-900, Brazil. E-mail: nespoli@ufmt.br

The ultrasonography (US) is a complementary technique of choice for the diagnostic of hepatic diseases in many domestics' species. In sheep however there are few reports about ultrasonography in hepatic diseases and there is not precise definition about the anatomic standards of normal liver limits in ultrasonographic examination. In this study 58 Santa Inês sheep breed were used and divided in 3 groups: $n 1=8$ males, $n 2=10$ not pregnant females and $n 3=40$ pregnant females. The animals were scanned from the $12^{\circ}$ to $8^{\circ}$ intercostal spaces (EI) to observe the localization of the vena cava caudal (VC), gallbladder (VB) and to measure the liver thickness above the VC and vena portae VP under the 11\% and $10^{\circ} \mathrm{EI}$. The liver was examined on satisfactory way from the $12^{\circ}$ till the $8^{\circ} \mathrm{El}$. Both the VC and the VP where observed from the $12^{\circ}$ to $9^{\circ} \mathrm{El}$, however the VC could not be observed in 11 animals, 10 of them were over $50 \mathrm{~kg}$. Between the two female groups the VC and VP where observed most frequently from the $11^{\circ}$ to $10^{\circ} \mathrm{El}$ and in all males examined from the $12^{\circ}$ to $10^{\circ} \mathrm{El}$. The location of the gallbladder varies between the $10^{\circ}$ to the $8^{\circ} \mathrm{El}$, with bigger incidence between the $9^{\circ}$ and the $8^{\circ} \mathrm{El}$ in pregnant and no pregnant females groups and underneath the $9^{\circ} \mathrm{EI}$ on the male group. Comparatively, the ecogenicity of the liver parenchyma was more intense than kidney cortex. There was a significant correlation between liver's weight and hepatic thikness above the vena portae on the $11^{\circ}$ and $10^{\circ} \mathrm{El}$ on the pregnant females group. The US supplied to important information about the topography and echogenicity of the liver and showed to be a useful tool to esteem the liver's weight.
\end{abstract}

INDEX TERMS: Sheep, ultrasonography, liver, Vena cava caudalis, Vena portae, gall bladder.

\footnotetext{
${ }^{1}$ Recebido em 15 de maio de 2008.

Aceito para publicação em 27 de fevereiro de 2009.

2 Departamento de Clínica Médica Veterinária, Faculdade Agronomia e Medicina Veterinária, Universidade Federal de Mato Grosso, Av.

Fernando Corrêa da Costa s/n, Coxipó, Cuiabá, MT 78060-900, Brasil.

*Autor para correspondência: nespoli@ufmt.br

${ }^{3}$ Departamento de Patologia e Clínica Veterinárias, Escola de Veterinária, Universidade Federal de Minas Gerais (UFMG), Av. Antônio Carlos 6627, Campus da UFMG, Belo Horizonte, MG 30123-970, Brasil.

${ }^{4}$ Depto Morfologia, Instituto de Ciências Biológicas, UFMG, Av. Antônio Carlos 6627, Campus Pampulha, Belo Horizonte, MG 31270-901.

5 Graduação em Medicina Veterinária, Escola de Veterinária, UFMG, Belo Horizonte, MG.

${ }^{6}$ Programa de Doutorado em Zootecnia, Escola de Veterinária, UFMG, Belo Horizonte, MG.

${ }^{7}$ Programa de Doutorado em Ciência Animal, Escola de Veterinária, UFMG, Belo Horizonte, MG.
}

RESUMO.- A ultra-sonografia (US) é uma das técnicas de exame complementar eletivas para o diagnóstico de enfermidades hepáticas de diversas espécies domésticas. Em ovinos, no entanto, existem poucos relatos sobre o aspecto ultra-sonográfico de enfermidades hepáticas e não há definição precisa dos padrões anatômicos da US normal do fígado. Neste estudo foram utilizados 58 ovinos da raça Santa Inês: $n 1=8$ machos, $n 2=10$ fêmeas não gestantes e n3=40 fêmeas gestantes. Os animais foram escaneados do $12^{\circ}$ ao $8^{\circ}$ espaços intercostais (EI) para se observar a localização da veia cava caudal (VC), veia porta (VP) e vesícula biliar (VB) e para se aferir a espessura do fígado sobre a VC e VP no $11^{\circ}$ e $10^{\circ} \mathrm{El}$. O fígado foi examinado de forma satisfatória do $12^{\circ}$ até o $8^{\circ}$ El. Nesta área, tanto a VC como a VP, foram observadas 
do $12^{\circ}$ ao $9^{\circ} \mathrm{EI}$, porém a VC não foi examinada de forma adequada em 11 animais, $10 \mathrm{com}$ peso acima de $50 \mathrm{~kg}$. Entre os dois grupos de fêmeas, a VC e a VP foram observadas com maior freqüência no $11^{\circ}$ e $10^{\circ} \mathrm{El}$ e em todos os machos examinados do $12^{\circ}$ ao $10^{\circ}$ El. A localização da vesícula biliar oscilou entre o $10^{\circ}$ e $\circ 8^{\circ} \mathrm{El}$, com maior incidência a nível do $9^{\circ}$ e $8^{\circ}$ EI nas fêmeas gestantes e não gestantes, e sobre o $9^{\circ} \mathrm{EI}$ nos machos. Comparativamente, a ecogenicidade do parênquima hepático foi mais intensa do que a do córtex renal. Houve correlação significativa entre o peso do fígado e a espessura hepática sobre a veia porta no $11^{\circ}$ e o $10^{\circ}$ El no grupo de fêmeas gestantes. A US forneceu informações importantes quanto a topografia e ecogenicidade do fígado e mostrou ser uma ferramenta útil para estimar o peso do órgão.

TERMOS DE INDEXAÇÃO: Ovinos, ultra-sonografia, fígado, veia cava caudal, veia porta, vesícula biliar.

\section{INTRODUÇÃO}

A ultra-sonografia (US) permite examinar de forma não invasiva a arquitetura do parênquima hepático, o sistema biliar e as veias hepáticas e portais (Braun 1990, Partington \& Biller 1995, Braun 1996). Em função destas características a US pode ser utilizada na investigação de uma variedade de enfermidades hepáticas (Nyland \& Park 1983), com utilidade particular naquelas hepatopatias que cursam com sinais clínicos inespecíficos e com resultados não conclusivos de patologia clínica (Braun 1996). Todavia, o método apresenta baixa especificidade, principalmente para doenças difusas, e o acesso a diagnósticos específicos requer frequentemente a realização de biópsias e de estudos histopatológicos (Partington \& Biller, 1995).

Apesar da aplicabilidade do método e do grande número de doenças que afetam o fígado, com destaque para as causas infecciosas, parasitárias, metabólicas e tóxicas (Pearson 2002), existem poucos artigos que abordem os aspectos ultra-sonográficos das enfermidades hepáticas nos ovinos. Os trabalhos disponíveis incluem o uso da ultrasonografia no estudo de alguns distúrbios com a hidatidose (Guarnera et al. 2001, Lahmar et al. 2007) e a fascilose (Gonzalo-Orden et al. 2003) e no diagnóstico isolado de neoplasia hepática (Lofstedt et al. 1988). Além disso, não há referência na literatura consultada de trabalhos básicos detalhados que envolvam o aspecto ultra-sonográfico do parênquima hepático, o acesso ao órgão e a localização das estruturas vasculares e da vesícula biliar nessa espécie.

Baseado nos fatos supracitados, o objetivo desse estudo foi estabelecer a melhor técnica de avaliação, janela sonográfica para análise do parênquima e suas principais estruturas vasculares e da vesícula biliar; determinar os padrões de ecogenicidade e morfológicos do parênquima e verificar a correlação entre medidas de profundidade do parênquima com o peso hepático total de ovinos.

\section{MATERIAL E MÉTODOS}

O experimento foi desenvolvido na Escola de Veterinária da Universidade Federal de Minas Gerais (UFMG), Belo Horizonte,
Brasil. Foram utilizados 58 ovinos da raça Santa Inês, subdivididos em três grupos: oito machos de seis a oito meses de idade $(20-30 \mathrm{~kg}), 10$ ovelhas adultas não gestantes $(41.99 \pm 7.23 \mathrm{~kg})$ e 40 ovelhas com 110-140 dias de gestação $(53,54 \pm 9.55 \mathrm{~kg})$. Os ovinos machos foram alocados em baias coletivas, alimentados com capim elefante picado e ração comercial e as fêmeas gestantes e não gestantes foram alimentadas com ração e feno de tifton.

Após tricotomia da metade caudal do gradil costal direito, contenção dos animais em decúbito lateral esquerdo e aplicação de gel para ultra-sonografia (Suprimed, Belo Horizonte), os espaços intercostais (EI) foram escaneados nos sentidos caudocranial e dorso-ventral, desde o $12^{\circ}$ até o $8^{\circ} \mathrm{EI}$, com aparelho de ultra-sonografia SSD-500V (Aloka, Japão) equipado com transdutor linear transretal de $5 \mathrm{MHz}$. Nestas varreduras foram observados 0 aspecto ultra-sonográfico do parênquima hepático e a melhor janela sonográfica da veia cava caudal (VC), veia porta (VP) e vesícula biliar (VB) de acordo com os El. Além disso, nos grupos de fêmeas gestantes e de não gestantes aferiu-se a espessura do fígado a partir da borda lateral das VC e VP no $11^{\circ}$ e $10^{\circ}$ El. Essas fêmeas foram abatidas cerca de dois dias depois e o peso do fígado sem a vesícula biliar foi mensurado (protocolo 77/2006 - Comitê de Ética em Experimentação Animal da UFMG).

O número total de identificações das estruturas vasculares (VC e VP) e da vesícula biliar em cada espaço intercostal examinado foi expresso em percentagem de acordo com a categoria animal avaliada. As medidas de espessura hepática (centímetros) foram correlacionadas com o peso total do fígado verificado após o abate dos animais, a partir da aplicação dos testes de correlação e regressão linear do programa BioEstat 4.0 (Ayres et al. 2005).

Para comparar as imagens observadas durante o exame ultra-sonográfico do fígado com as estruturas anatômicas correspondentes, procedeu-se a fixação por formaldeído a 10\% de uma borrega (Santa Inês) de 26 kg e aplicação de látex azul nas veias safena lateral direita e mesentérica cranial de acordo com Bradbury \& Hoshino (1978). Após secagem à sombra, a peça foi seccionada em fatias transversais, no sentido dos espaços intercostais, desde o $12^{\circ}$ até o $8^{\circ} \mathrm{El}$. As fotografias do fígado e das estruturas adjacentes obtidas das superfícies seccionadas sobre cada espaço intercostal foram comparadas com as imagens ultra-sonográficas provenientes do mesmo animal antes da eutanásia e dos demais animais utilizados no experimento.

\section{RESULTADOS}

O fígado foi detectado em todos os animais do $11^{\circ}$ ao $8^{\circ}$ El com o equipamento e método utilizados. A margem caudal do órgão foi visibilizada no $12^{\circ}$ El em todos os machos, em $40 \%$ das fêmeas vazias e em $27,5 \%$ das fêmeas gestantes. Neste espaço verificou-se desde uma pequena porção dorso-caudal do fígado, com eventual sobreposição ao rim direito, até visibilização ampla do parênquima hepático e das veias cava caudal e porta. Nos demais animais examinados o fígado foi observado a partir do11으. $\mathrm{El}$.

Do $12^{\circ}$ ao $10^{\circ}$ El houve pouca interferência do pulmão direito sobre a formação da imagem hepática. A partir do $9^{\circ} \mathrm{El}$, entretanto, a parte dorsal do parênquima hepático e 


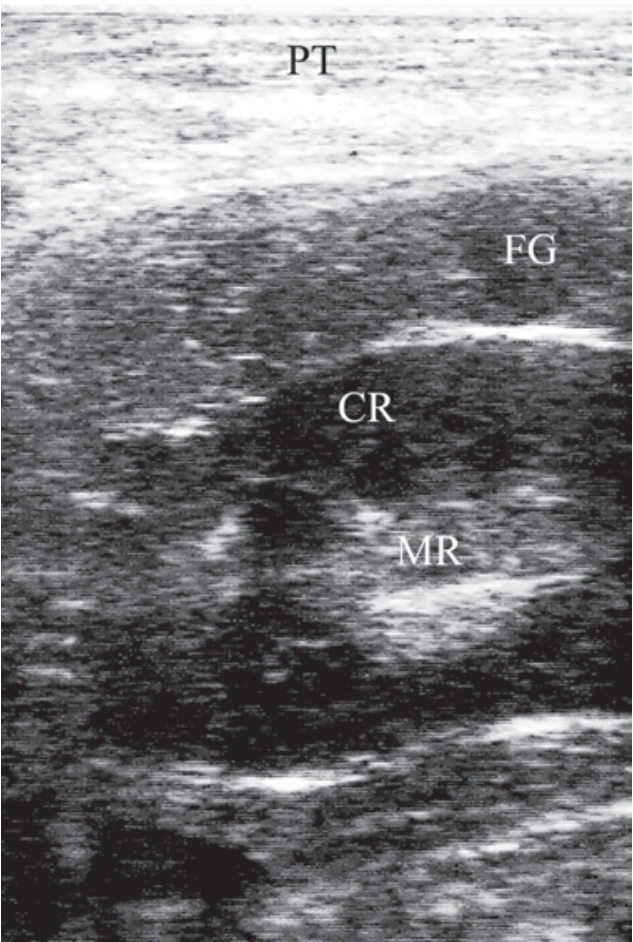

Fig.1. Ultra-sonografia linear do fígado e do rim direito no $12^{\circ}$ espaço intercostal (transdutor de $5 \mathrm{MHz}$ ). PT: parede torácica, FG: fígado, CR: córtex renal, MR: medular renal. a veia cava caudal situaram-se sob o pulmão direito e só puderam ser examinadas nos animais menores durante a expiração. No 8ํㅡㄹ 0 acesso ultra-sonográfico se restringiu à margem ventral do órgão, com maior alcance também na fase expiratória da respiração. A partir da comparação desta área de projeção ultra-sonográfica com a peça anatômica verificou-se acesso ultra-sonográfico amplo sobre os lobos direito e caudado, mas limitado sobre o lobo esquerdo, que ficou sobreposto pelos pulmões em praticamente toda a sua extensão, exceto na sua margem ventral.

O padrão ultra-sonográfico do parênquima hepático consistiu de numerosos ecos fracos distribuídos de forma homogênea pelo parênquima. Na varredura do fígado e rim direito no mesmo plano, o córtex renal apresentou nitidamente menor ecogenicidade do que o parênquima hepático (Fig.1). As veias cava caudal e porta apareceram com lúmen anecóico e limites bem definidos, porém diferente da veia cava e veias hepáticas a parede dos vasos portais apresentaram bordas ecogênicas, sobretudo nos vasos de maior calibre (Fig.2 e 3). A imagem da vesícula biliar se caracterizou por formato predominantemente piriforme, com lúmen anecóico e paredes ecogênicas.

A veia cava caudal, localizada na porção dorso-medial do fígado, apresentou formato predominantemente elíptico (Fig.2D) nas varreduras transversais e foi detectada do $12^{\circ}$ ao $9^{\circ}$ El em todas as categorias de animais avaliados
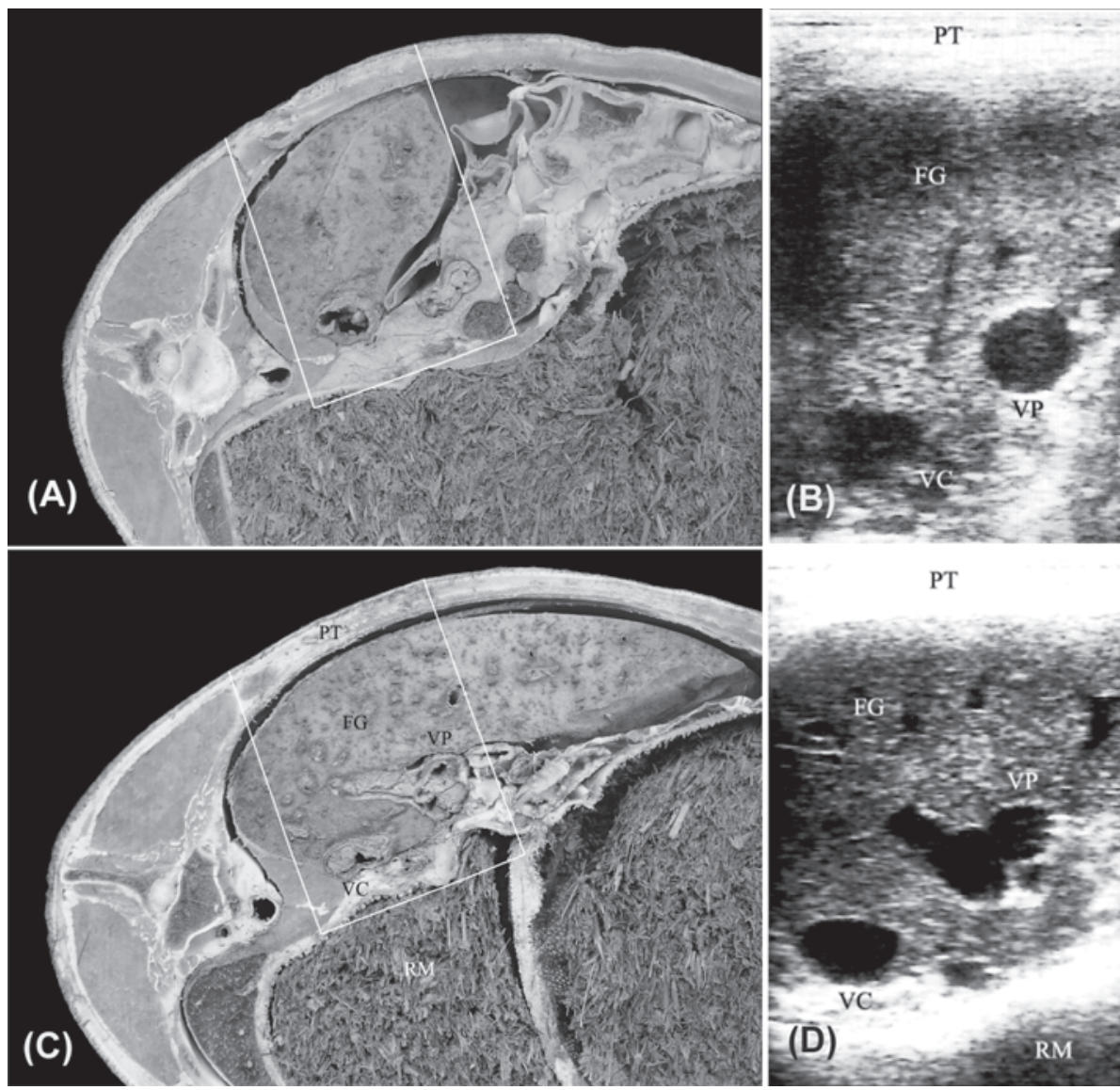

Fig.2. Espécie ovina. Peça anatômica fixada com formol $10 \%$ e injetada com látex azul nas veias safena e mesentérica cranial. (A) Corte transversal ao longo do $12^{\circ} \mathrm{El}$. Área em destaque (retângulo branco) corresponde à imagem ultra-sonográfica ao lado. (B) Ultrasonografia linear do fígado no $12^{\circ} \mathrm{El}$ (transdutor de $5 \mathrm{MHz}$ ). (C) Corte transversal ao longo do $11^{\circ} \mathrm{El}$. Área em destaque (retângulo branco) corresponde à imagem ultra-sonográfica ao lado. (D) Ultra-sonografia linear do fígado no $11^{\circ}$ El (transdutor de $5 \mathrm{MHz}$ ). PT = parede torácica, $\mathrm{FG}$ = fígado, $\mathrm{VC}=$ veia cava caudal, $\mathrm{VP}=$ veia porta, $\mathrm{RM}=$ rúmen . 
Quadro 1. Percentual de observação ultra-sonográfica da veia cava caudal de acordo com o espaço intercostal

\begin{tabular}{lcccc}
\hline \multicolumn{1}{c}{ El / animais } & $12^{\circ}$ & $11^{\circ}$ & $10^{\circ}$ & $9^{\circ}$ \\
\hline Machos (8) & 100 & 100 & 100 & 37,5 \\
Não gestantes (9) & 33,33 & 100 & 100 & 55,56 \\
Gestantes (30) & 13,33 & 100 & 96,67 & 43,33
\end{tabular}

Quadro 2. Percentual de observação ultra-sonográfica da veia porta de acordo com o espaço intercostal

\begin{tabular}{lcccc}
\hline \multicolumn{1}{c}{ El / animais } & $12^{\circ}$ & $11^{\circ}$ & $10^{\circ}$ & $9^{\circ}$ \\
\hline Machos (8) & 100 & 100 & 100 & 75 \\
Não gestantes (10) & 20 & 100 & 100 & 80 \\
Gestantes (40) & 15 & 97,5 & 100 & 75
\end{tabular}

Quadro 3. Percentual de observação ultra-sonográfica da vesícula biliar de acordo com o espaço intercostal

\begin{tabular}{lccc}
\hline \multicolumn{1}{c}{ El / animais } & $10^{\circ}$ & $9^{\circ}$ & $8^{\circ}$ \\
\hline Machos (8) & 37,5 & 87,5 & 12,5 \\
Não gestantes (10) & 20 & 90 & 90 \\
Gestantes (40) & 30 & 77,5 & 75
\end{tabular}

Quadro 4. Percentual de observação ultra-sonográfica da vesícula biliar em um ou mais espaço intercostal

\begin{tabular}{lccc}
\hline No de El / animais & 1 & 2 & 3 \\
\hline Machos (8) & 62,5 & 37,5 & - \\
Não gestantes (10) & 20 & 60 & 20 \\
Gestantes (40) & 40 & 52,5 & 7,5
\end{tabular}

Quadro 5. Melhor janela sonográfica (EI) para observação da veia cava, veia porta e vesícula biliar de acordo com as categorias de animais

\begin{tabular}{llcc}
\hline \multirow{2}{*}{ Animais } & \multicolumn{3}{c}{ Estruturas examinadas } \\
\cline { 2 - 4 } & Veia cava & Veia porta & Vesícula biliar \\
\hline Machos (8) & $12^{\circ}$ ao $10^{\circ}$ & $12^{\circ}$ ao 9o & $9^{\circ}$ \\
Não gestantes (10) & $11^{\circ}$ ao $10^{\circ}$ & $11^{\circ}$ ao $9^{\circ}$ & $9^{\circ}$ ao $8^{\circ}$ \\
Gestantes (40) & $11^{\circ}$ ao $10^{\circ}$ & $11^{\circ}$ ao $9^{\circ}$ & $9^{\circ}$ ao $8^{\circ}$
\end{tabular}

(Fig.2 e 3). Entretanto, houve dificuldade em identificar o vaso com o transdutor de $5 \mathrm{MHz}$ em 11 animais, a maior parte gestante, com peso vivo e escore corporal acima de $50 \mathrm{~kg} \mathrm{e} \mathrm{4,} \mathrm{respectivamente.} \mathrm{Afora} \mathrm{estes} \mathrm{casos,} \mathrm{a} \mathrm{melhor}$ janela sonográfica para identificação da VC entre os diferentes grupos foi sobre a porção dorsal do $11^{\circ}$ e o $10^{\circ} \mathrm{EI}$ (Quadros 1 e 5).

A veia porta, com forma invariavelmente arredondada, foi identificada em todos os animais em uma posição ventral e levemente lateral à veia cava, independente da categoria e do peso dos animais examinados (Fig.2 e 3). A observação da imagem da VP também se estendeu do $12^{\circ}$ ao $9^{\circ} \mathrm{El}$, porém, a melhor janela acústica observada desse vaso foi mais ampla que a da VC e incluiu o $11^{\circ}$, $10^{\circ}$ e o 9 l (Quadros 2 e 5).

A localização da vesícula biliar oscilou entre o $10^{\circ}$ e 0 8 l (Fig.3). Nas fêmeas gestantes e não gestantes, a melhor janela acústica para a visibilização da VB foi iden-

Fig.3. Espécie ovina. Peça anatômica fixada com formol $10 \%$ e injetada com látex azul nas veias safena e mesentérica cranial. (A) Corte transversal ao longo do $10^{\circ} \mathrm{El}$. Área em destaque (retângulo branco) corresponde à imagem ultra-sonográfica ao lado. (B) Ultrasonografia linear do fígado no $10^{\circ} \mathrm{El}$ (transdutor de $5 \mathrm{MHz}$ ). (C) Corte transversal ao longo do 9 El. Área em destaque (retângulo branco) corresponde à imagem ultra-sonográfica ao lado. (D) Ultra-sonografia linear do fígado no 9o El (transdutor de $5 \mathrm{MHz}$ ). $\mathrm{PT}=$ parede torácica, PD = pulmão direito, FG = fígado, $\mathrm{VC}=$ veia cava caudal, $\mathrm{VPE}=$ ramo esquerdo da veia porta, $\mathrm{VH}=$ veia hepática, VB = vesícula biliar, $\mathrm{RM}=$ rúmen, $\mathrm{OM}$ = omaso.
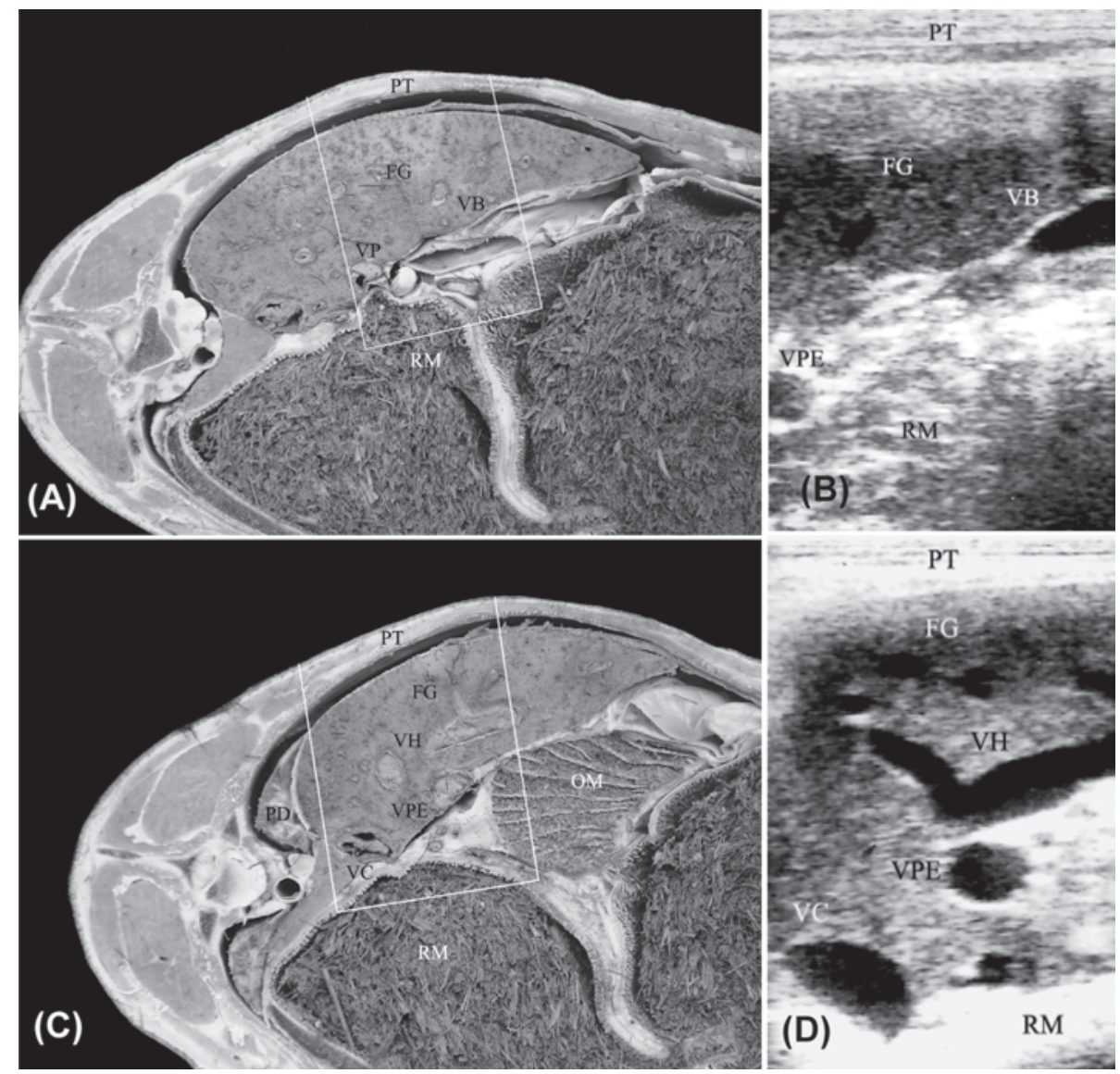


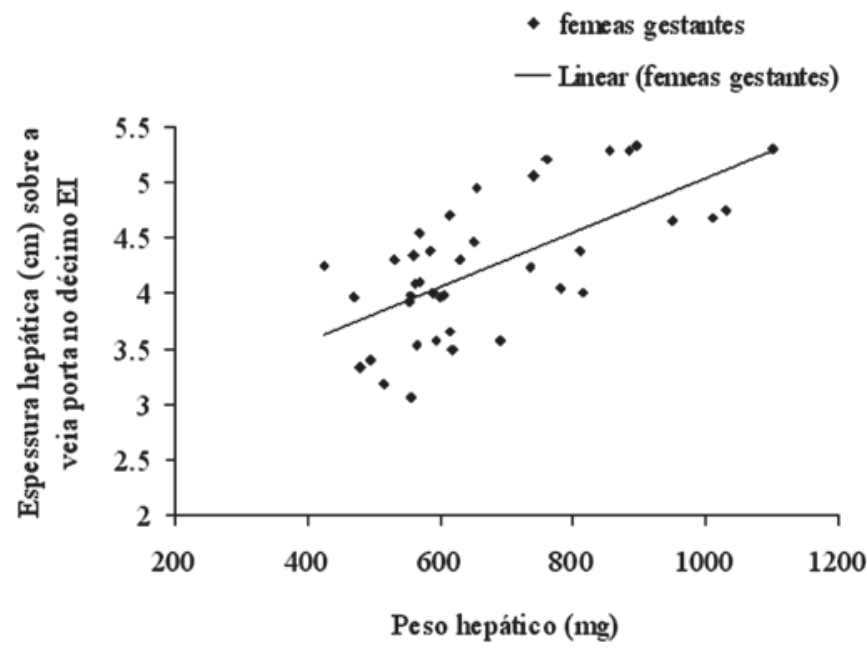

Fig.4. Correlação entre o peso do fígado dos animais com a espessura hepática a partir da margem lateral da veia porta no $11^{\circ} \mathrm{EI}(p<0.0001$ e $R=0,659)$.

\section{Quadro 6. Correlação entre o peso hepático total com a espessura do fígado sobre a VC e a VP no $11^{\circ}$ e $10^{\circ}$ espaço intercostal}

\begin{tabular}{|c|c|c|c|c|}
\hline $\begin{array}{c}\text { Animais/ } \\
\text { (№ ) }\end{array}$ & Vaso/ El & (p) & $\mathrm{R} \%$ & $\begin{array}{l}\text { Equação de } \\
\text { regressão }\end{array}$ \\
\hline $\mathrm{ONG}^{\mathrm{a}}(9)$ & $\mathrm{VC} / 11^{\circ}$ & 0,18 & - & - \\
\hline ONG (9) & $\mathrm{VC} / 10^{\circ}$ & 0,76 & - & - \\
\hline ONG (10) & VP/110 & 0,7 & - & - \\
\hline ONG (10) & VP/10 $10^{\circ}$ & 0,85 & - & - \\
\hline $\mathrm{OG}^{\mathrm{b}}(30)$ & $\mathrm{VC} / 11^{\circ}$ & 0,13 & - & - \\
\hline OG (29) & $\mathrm{VC} / 10^{\circ}$ & 0,53 & - & - \\
\hline OG (39) & $\mathrm{VP} / 11^{\circ}$ & 0,0042 & 44,8 & $Y^{C}=134,292+119,183 X^{d}$ \\
\hline OG (39) & VP/10 & $<0,0001$ & 65,9 & $Y=-81,402+177,926 X$ \\
\hline
\end{tabular}

a Ovelhas não gestantes, b Ovelhas gestantes, ${ }^{c}$ Peso estimado do fígado, ${ }^{d}$ Espessura do fígado em centímetros.

tificada sobre o 9 e o 8 $\mathrm{El}$, sob a margem ventro-medial do fígado. Enquanto, entre os machos, a maioria das identificações desta estrutura ocorreu sobre o 9 El (Quadro 3 e 5). A VB foi identificada com maior freqüência em apenas um El em machos e em dois El nas fêmeas gestantes e não gestantes (Quadro 4).

Houve correlação significativa entre o peso do fígado e a espessura hepática a partir da superfície lateral da veia porta no $11^{\circ}$ e no $10^{\circ}$ El para o grupo de fêmeas gestantes (Quadro 6 e Fig.4). Para as demais análises os resultados obtidos não foram significativos, seja para a espessura hepática sobre a veia porta nas fêmeas não gestantes ou nas demais mensurações sobre a veia cava caudal.

\section{DISCUSSÃO E CONCLUSÕES}

Ficou demonstrado que o fígado pode ser escaneado em ovinos desde $012^{\circ}$ até $08^{\circ}$ El. Os achados ultrasonográficos observados correspondem em grande parte com as descrições de artigos semelhantes realizados em bovinos (Yamaga \& Too 1984, Braun 1990, Braun \& Geber 1994) e caprinos (Yamaga \& Too 1984). Em bovinos, o fígado pode ser examinado de maneira similar, do $12^{\circ}$ até o 8 El (Braun \& Geber 1994), o padrão ecogênico do parênquima hepático, das estruturas vasculares e da vesícula biliar é muito parecido e a avaliação ultrasonográfica do órgão também está sujeita à interferência do pulmão direito a partir do 9ํㅡ El (Braun 1990).

Entretanto, no presente estudo, a VC e a VP foram identificadas em um pequeno percentual das fêmeas examinadas no $12^{\circ} \mathrm{El}$, sobretudo naquelas fêmeas gestantes, diferente dos achados relatados por Braun \& Geber (1994) em bovinos (Quadros 1 e 2). Este fato pode ser explicado pelo menos em parte pelo posicionamento lateral em que o animal foi examinado e pela condição de gestação, que provavelmente favoreceram o deslocamento do órgão no sentido cranial. Entre os ovinos machos, no entanto, ambas as veias foram identificadas no $12^{\circ} \mathrm{El}$ em todos os animais examinados. Outro ponto divergente foi a diferença observada na visibilização da VC nos EI mais craniais como $10^{\circ}$ e 9 El. A VC foi identificada em praticamente todos os casos no $10^{\circ} \mathrm{El}$ e em percentual considerável no $9^{\circ} \mathrm{El}$, em todas as categorias dos ovinos estudados (Quadro 1). Em contrapartida, nos bovinos, a VC tem sido observada em apenas $21,5 \%$ das vezes no $10^{\circ}$ El (Braun \& Geber 1994) e não pôde ser visualizada no $9^{\circ} \mathrm{El}$ em virtude da sobreposição do pulmão direito com o fígado (Braun 1990, Braun \& Geber 1994).

Além disso, a vesícula biliar (VB), localizada entre $011^{\circ}$ e 9 El nos bovinos (Braun 1996), foi observada nos ovinos entre $010^{\circ}$ e o $8^{\circ} \mathrm{El}$, inclusive no grupo dos machos que apresentaram imagem hepática a partir do $120 \mathrm{E}$. De qualquer forma, não há como afirmar que existam realmente diferenças entre as duas espécies neste quesito, já que os estudos com bovinos foram conduzidos com os animais em estação e não pode-se descartar a influência do posicionamento sobre um possível deslocamento da VB.

A ecogenicidade do parênquima observada correspondeu aos padrões descritos para bovinos e caprinos (Yamaga \& Too 1984, Braun 1990, Braun \& Geber 1994), inclusive no que se refere às diferenças entre o parênquima hepático e 0 córtex renal (Fig.1) apontadas em estudos em bovinos (Acorda et al. 1994a), caninos (Partington \& Biller 1995) e felinos (Yager \& Mohammed 1992). No presente estudo, assim como em bovinos (Acorda et al. 1994a), houve dificuldade em muitos casos de se escanear o fígado e o rim direito sob o mesmo plano, provavelmente em função do tipo de transdutor utilizado. Como a superfície disponível é pequena e limitada pelas costelas, provavelmente um transdutor setorial, com menor área de contato forneceria imagens simultâneas de ambos os órgãos com menor dificuldade.

O uso deste contraste entre a ecogenicidade hepática e renal é importante para o diagnóstico de uma variedade de doenças difusas do parênquima hepático freqüentes nesta espécie, que cursam essencialmente com aumento ou diminuição da ecogenicidade. Entre as que determinam aumento da ecogenicidade estão inclusas as lipidoses hepáticas (Braun et al. 1996), as intoxicações crônicas por plantas (Braun et al. 1999) e as distrofias 
hepáticas (Acorda et al. 1994b). Já entre as que tornam o fígado hipoecóico figuram as doenças degenerativas (Acorda et al. 1995) e os processos de congestão de origem cardíaca (Braun et al. 1996).

Da mesma forma, o conhecimento da localização e do aspecto ultra-sonográfico das veias e da vesícula biliar, fornecem subsídios importantes para o diagnóstico de alterações vasculares e de afecções que afetam o sistema biliar. Apesar dos ductos intra-hepáticos não serem distinguíveis através da ultra-sonografia, possíveis alterações como distensão e calcificação das suas paredes podem ser identificadas paralelamente aos vasos portais, já que tais ductos acompanham o curso dos ramos da veia porta (Habel, 1986). A ultra-sonografia tem se mostrado útil nos casos de fasciolose hepática em ovinos, para a detecção dos trematódeos na luz dilatada dos ductos biliares (GonzaloOrden et al. 2003) e pode ser utilizada no diagnóstico de processos obstrutivos e em infecções do trato biliar, de maneira similar àquela descrita para bovinos (Braun et al. 1995).

Apesar do uso do transdutor de $5 \mathrm{MHz}$ não ter possibilitado a avaliação adequada da porção dorso-caudal do fígado e da veia cava em 11 animais, esta freqüência de transdutor foi eficaz em avaliar a grande maioria dos animais e permitiu exame completo dos vasos portais e da vesícula biliar em todos os ovinos avaliados. Entretanto, para os animais acima de $50 \mathrm{~kg}$, com escore corporal acima de 4, o exame completo do órgão em alguns animais pode depender de transdutores com freqüências mais baixas, como as de $3,5 \mathrm{MHz}$.

A correlação significativa entre o peso hepático e as medidas da espessura do fígado apontada neste estudo tem sido relatada em avaliações correspondentes em bovinos (Braun et al. 1994). No entanto, nos bovinos as correlações significativas não ficaram restritas às aferições sobre a VP, mas ocorreram também nas comparações sobre a VC, com coeficientes de correlação mais expressivos, a maior parte situados entre 67 e $76 \%$. Entre os trabaIhos realizados em caninos os resultados observados foram variáveis (Godshalk et al. 1988, Barr 1992). Barr (1992) observou correlações significativas entre mensurações transversais e longitudinais do fígado, com coeficiente de correlação de 85\%, enquanto Godshalk et al. (1988) identificaram correlação significativa apenas em um tipo de aferição realizada, com coeficiente de correlação de $51 \%$. A ausência de correlação observada entre as fêmeas não gestantes neste estudo, assim como por Godshalk et al. (1988) pode ter relação com o menor número de animais utilizados em ambos os trabalhos, 10 e 16 respectivamente. De qualquer forma, em virtude da agilidade do procedimento e da correlação observada, as mensurações do parênquima hepático sobre a veia porta propiciam índices úteis para estimar o tamanho do fígado de ovinos.

\section{REFERÊNCIAS}

Acorda J.A., Yamada H. \& Ghamsari S.M. 1994a. Ultrasonography of fatty infiltration of the liver in dairy cattle using liver-kidney contrast. Vet. Radiol. Ultrasound 35:400-404.
Acorda J.A., Yamada H. \& Ghamsari S.M. 1994b. Ultrasonographic features of diffuse hepatocellular disorders in dairy cattle. Vet. Radiol. Ultrasound 35:196-200.

Acorda J.A., Yamada H. \& Ghamsari S.M. 1995. Comparative evaluation of hydropic degeneration of the liver in dairy cattle biochemistry, ultrasonography and digital analysis. Vet. Radiol. Ultrasound 36(4):322326.

Ayres M., Ayres M.J., Ayres D.L. \& Santos A.A.S. 2005. Bioestat: aplicações estatísticas nas áreas das ciências biomédicas. Belém: Mamirama. Disponível <www.mamirama.org.br> Acesso em 25 set. 2007.

Bradbury S.A. \& Hoshino K. 1978. An improved embalming procedure forlong-lasting preservation of the cadaver for anatomical study. Acta Anatomica.101:97-103

Barr F. 1992. Ultrasound assesment of liver in the dog. J. Small Anim. Pract. 33:359-364.

Braun U. 1990. Ultrasonographic examination of the liver in cows. Am. J. Vet. Res. 51(10):1522-1526.

Braun U. \& Geber D. 1994. Influence of age, breed, and stage of pregnancy on hepatic ultrasonographic findings in cows. Am. J. Vet. Res. 55(9):1201-1205.

Braun U., Pospischil A., Pusterla N. \& Winder C. 1995. Ultrasonographic findings in cows with cholestasis. Vet. Rec. 137(21):537-43.

Braun U. 1996. Ultrasonographic examination of the liver and gall bladder in cows: abnormal findings. Comp. Contin. Educ. 18(2):s61-s73.

Braun U., Pusterla N. \& Wild K. 1996. Ultrasonographic examination of the liver and gallbladder in cows: abdnormal findings. Comp. Contin. Educ. 18(11):1255-1269.

Braun U., Linggi T. \& Pospischil A. 1999. Ultrasonographic findings in three cows with chronic ragwort (Senecio alpinus) poisoning. Vet. Rec. 144(5):122-126.

Godshalk C.P., Badertscher R.R. \& Rippy M.K. 1988. Quantitative ultrasonic assessment of liver size in the dog. Vet. Radiol. 29:162-167.

Gonzalo-Orden M., Millán L., Álvarez M., Sánchez-Campos S., Jiménes R., Gonzáles-Gallego J. \& Tuñón M.J. 2003. Diagnostic imaging in sheep hepatic fascioliasis: ultrasound, computer tomography and magnetic resonance findings. Parasitol. Res. 90:359-364.

Guarnera E.A., Zanzottera E.M., Pereyra H. \& Franco A.J. 2001. Ultrasonographic diagnosis of ovine cystic echinococcosis. Vet. Radiol. Ultrasound 42(4):352-354.

Habel R.E. 1986. Sistema digestivo, p.807-858. In: Getty R. (Ed.), Anatomia dos Animais Domésticos. $3^{\underline{a}}$ ed. Guanabra Koogan, Rio de Janeiro.

Lahmar S., Ben Chéhida F., Pétavy A.F., Hammou A., Lahmar J., Ghannay A., Gharbi H.A. \& Sarciron M.E. 2007. Ultrasonographic screening for cystic echinococcosis in sheep in Tunisia. Vet. Parasitol. 143:42-49.

Lofstedt J., Schelling S., Stowater J. \& Morris E. 1988. Antemortem diagnosis of hepatic adenocarcinoma in a ewe. J. Am. Vet. Med. Assoc. 193(12):1537-1538.

Nyland T.G. \& Park R.D. 1983. Hepatic ultrasonography in the dog. Vet. Radiol. 24:74-84.

Partington B.P. \& Biller D.S. 1995. Hepatic imaging with radiology and ultrasound. Vet. Clin. North Am., Small Anim. Pract. 25(2):305-335.

Pearson E.G. 2002. Diseases of the hepatobiliary system, p.790-824. In: Smith B.P. (Ed.), Large Animal Internal Medicine. 3rd ed. Mosby, St Louis.

Yager A.E. \& Mohammed H. 1992. Accuracy of ultrasonography in the detection of severe hepatic lipidosis in cats. Am. J. Vet. Res. 53(4):597599.

Yamaga Y. \& Too K. 1984. Diagnostic ultrasound imaging in domestic animals: fundamental studies on abdominal organs and fetuses. Jpn. J. Vet. Sci. 46(2):203-212. 